\title{
Prognostic value of Neutrophil-to-lymphocyte ratio in COVID-19 patients: A systematic review and meta-analysis
}

\author{
Juan R. Ulloque-Badaracco ${ }^{1}$, Ivan Salas-Tello ${ }^{1}$, Ali Al-kassab-Cordova ${ }^{1}$, Esteban A. \\ Alarcon-Braga ${ }^{1}$, Vicente Benites-Zapata ${ }^{2}$, Jorge Maguiña ${ }^{1}$, and Adrian V. Hernandez ${ }^{2}$ \\ ${ }^{1}$ Universidad Peruana de Ciencias Aplicadas \\ ${ }^{2}$ Universidad San Ignacio de Loyola
}

February 1, 2021

\begin{abstract}
Background: Neutrophil-to-lymphocyte ratio (NLR) is an accessible and widely used biomarker. NLR may be used as an early marker of poor prognosis in patients with COVID-19. Methods: We conducted a systematic review and meta-analysis. Observational studies that reported the association between baseline NLR values (i.e. at hospital admission) and severity or all-cause mortality in COVID-19 patients were included. The quality of the studies was assessed using the Newcastle-Ottawa scale (NOS). Random effects models and inverse variance method were used for meta-analyses. The effects were expressed as odds ratios (OR) and their 95\% confidence intervals (CI). Small study effects were assessed with the Egger's test. Results: Twenty studies, 19 cohorts and one case-control were included. An increase of one unit of NLR was associated with a higher odds of COVID-19 severity (OR 6.6, 95\% CI: $4.71-7.19 ; \mathrm{p}<0.001$ ) and higher odds of all-cause mortality (OR 12.7, 95\% CI: $1.32,123.36 ; \mathrm{p}=0.025)$. No differences were found in subgroup analyses by study design. The subgroup analysis of the studies, by country of origin, showed that the strength of the association between NLR and mortality was greater in Chinese studies (OR 31.1; 95\%CI 19.57 to $49.3 ; \mathrm{p}<0.0001$ ) with moderate heterogeneity (I2 =43\%). In our sensitivity analysis, we found that 7 studies with low risk of bias maintained strong association between both outcomes and the NLR values (severity: OR 4.7; 95\% CI 3.5 to $6.34 ; \mathrm{p}<0.001$ vs mortality: OR $31.1 ; 95 \%$ CI 19.57 to $49.3 ; \mathrm{p}<0.0001$ ), with low (I2 = 37\%) and moderate (I2 = $43 \%$ ) heterogeneity for severity and mortality outcomes, respectively. No publication bias was found for studies that evaluated effects for the severity of disease. Conclusions: Higher values of NLR were associated with severity and all-cause mortality in hospitalized COVID-19 patients.
\end{abstract}

Prognostic value of Neutrophil-to-lymphocyte ratio in COVID-19 patients: A systematic review and meta-analysis

Running title: Prognostic value of NLR in COVID-19 patients

Juan R. Ulloque-Badaracco ${ }^{1}$, W. Ivan Salas-Tello ${ }^{1}$, Ali Al-kassab-Córdova ${ }^{1}$, Esteban A. Alarcón-Braga ${ }^{1}$, Vicente A. Benites-Zapata ${ }^{2}$, Jorge L. Maguiña ${ }^{1}$ Adrian V.Hernandez ${ }^{3,4}$

\section{Filiation:}

${ }^{1}$ Escuela de Medicina, Universidad Peruana de Ciencias Aplicadas, Lima, Peru.

${ }^{2}$ Vicerrectorado de Investigación, Universidad San Ignacio de Loyola (USIL), Lima, Peru

${ }^{3}$ Unidad de Revisiones sistemáticas y Meta-análisis, Guías de Práctica Clínica y Evaluaciones de Tecnología Sanitaria (URSIGET), Vicerrectorado de Investigación, Universidad San Ignacio de Loyola (USIL), Lima, Peru. 
${ }^{4}$ Health Outcomes, Policy, and Evidence Synthesis (HOPES) Group, University of Connecticut School of Pharmacy

\section{Corresponding author:}

Jorge L. Maguiña

Escuela de Medicina, Universidad Peruana de Ciencias Aplicadas, Lima, Peru

Av. Alameda San Marcos, Distrito de Chorrillos 15067. Lima, Peru.

Telephone: +5116303333

E-mail: jorge.luis.maguina@gmail.com

Acknowledgments: None. No funding to declare.

Conflicts of interest : No conflicts of interest for all authors.

\section{Authors Contributions}

Study design and concept: IST, JRUB, JLM, AVH, VB-Z. Acquisition of data: AAC, EA-B, JRUB, IST, AVH, VB-Z, JLM. Drafting of the manuscript: JRUB, IST, AAC, EA-B. Critical revision of the manuscript: AVH, VB-ZM, JLM, Statistical analysis: VB-ZM, JRUB, IST, AVH. Study supervision: AVH, JLM, VB-Z.

\section{Hosted file}

Prognostic value of Neutrophil-to-lymphocyte ratio in COVID-19 patients IJCP.pdf available at https://authorea.com/users/393096/articles/506808-prognostic-value-of-neutrophil-tolymphocyte-ratio-in-covid-19-patients-a-systematic-review-and-meta-analysis 
FIGURE 1 : PRISMA 2009 Flow Diagram

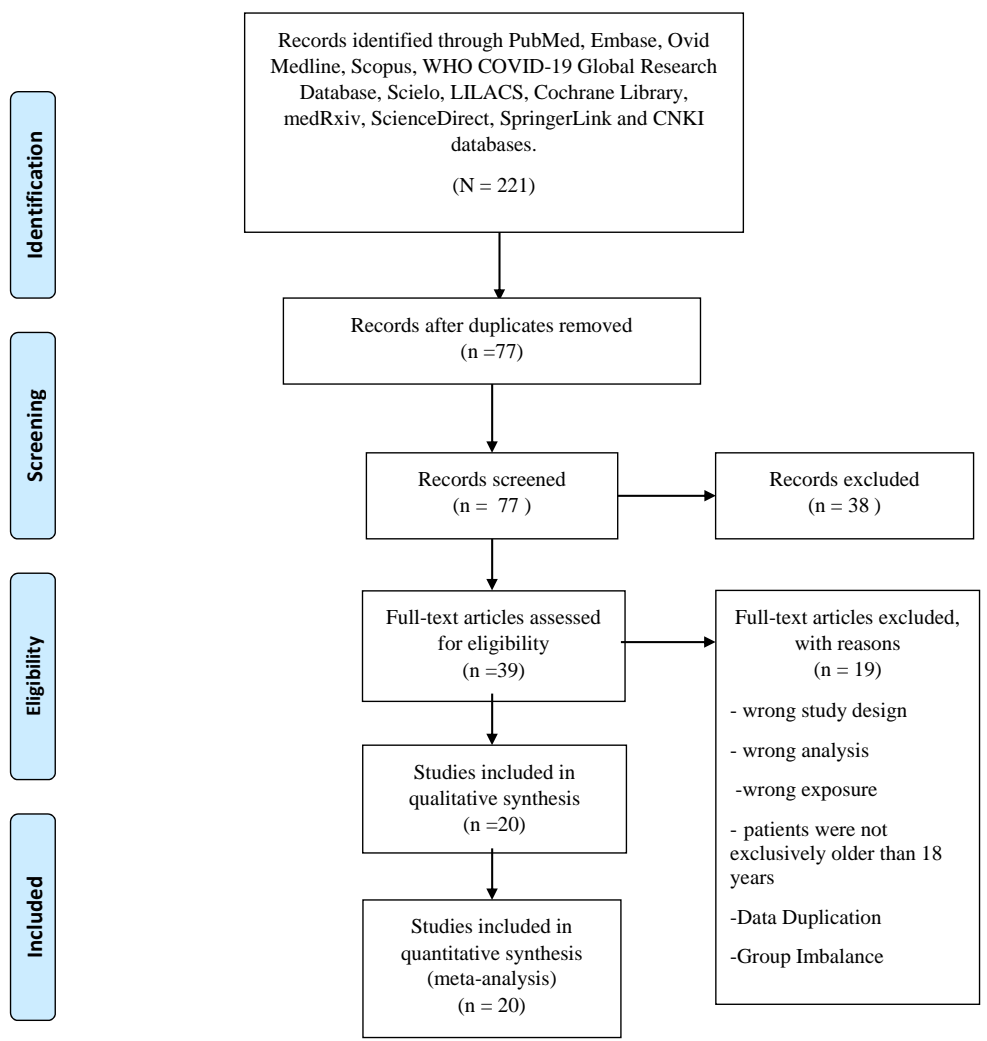


Figure 2: Association between NLR and severity

Figure 2A. Forest Plot for studies that evaluated the association of NLR and severity

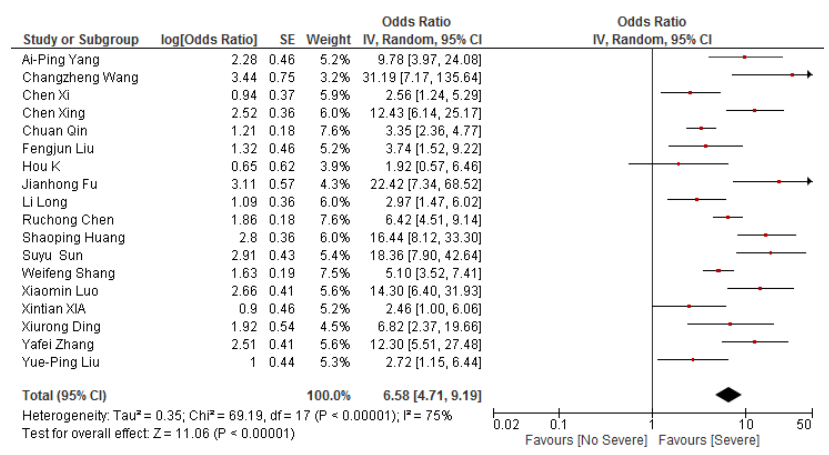


Figure 2B. Subgroup analysis according to study design of the studies that evaluated the association between NLR and severity

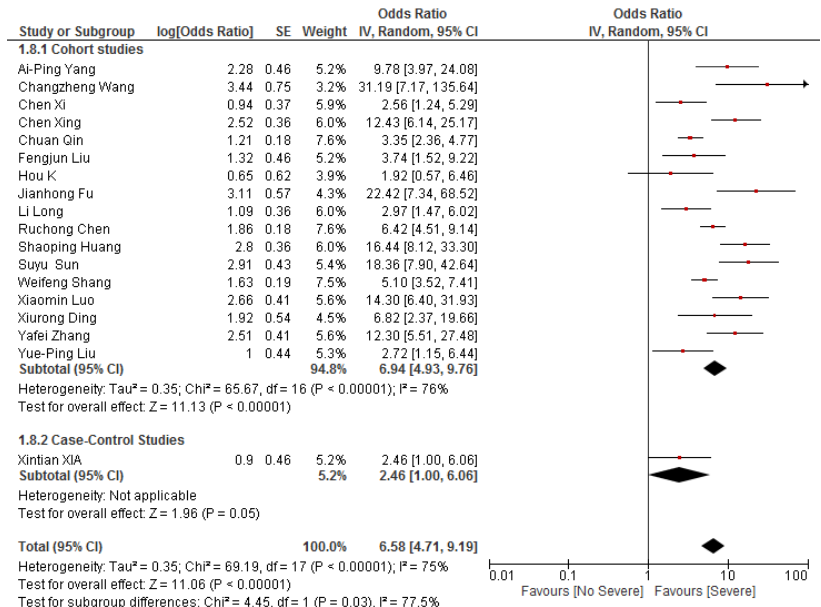

Test for overall entect $z=11.06(P<0.00001)$

Favours [No Severe] Favours [Severe] 
Figure 2C. Sensitivity analysis according to the risk of bias of the studies that evaluated the association between NLR and severity

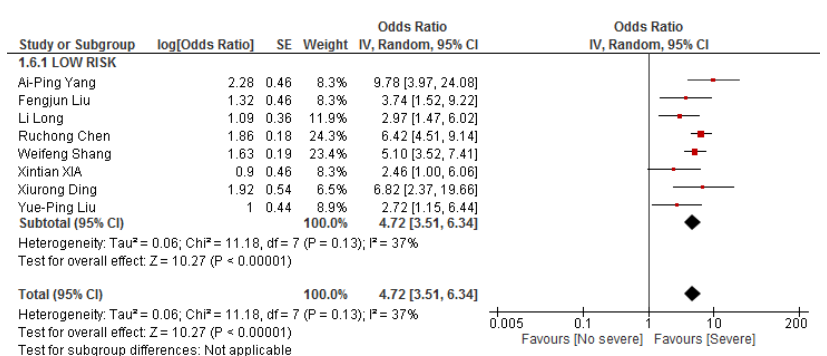


Figure 3: Association between NLR and mortality

Figure 3A. Forest Plot for studies that evaluated the association between NLR and mortality

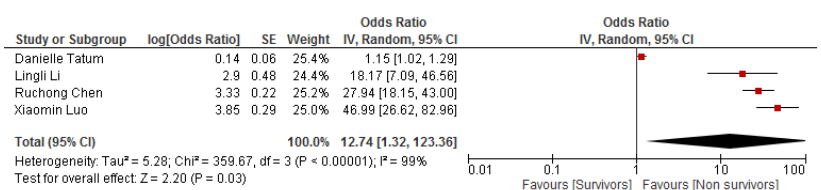

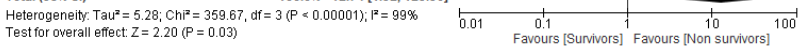


Figure 3 B: Subgroup analysis according to origin country of the studies that evaluated the association between NLR and mortality

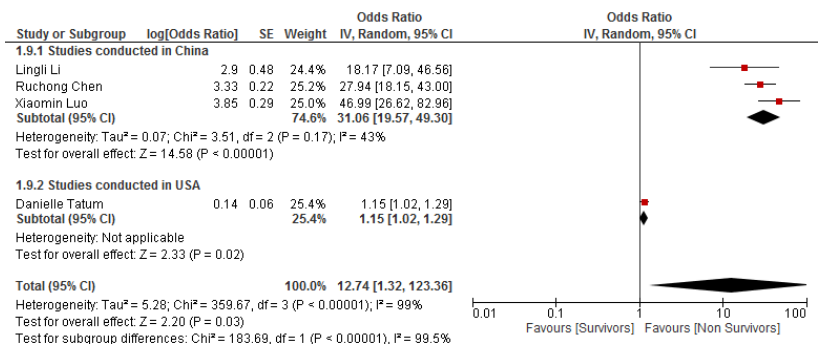


Figure 3C: Sensitivity analysis according to the risk of bias of the studies that evaluated the association between NLR and mortality

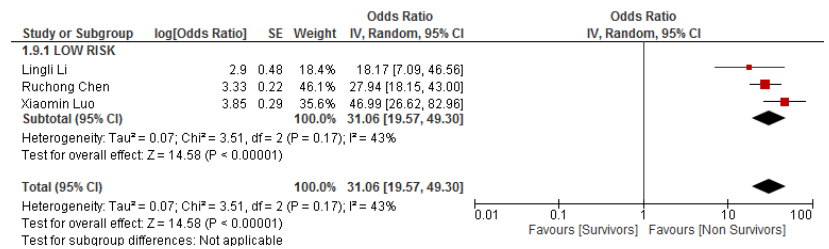

